\title{
Evaluating the Impact of Tablet Computers on Design Engineering
}

\author{
Remon Pop-Iliev, Scott Nokleby and George Platanitis \\ University of Ontario Institute of Technology \\ remon.pop-iliev@uoit.ca \\ scott.nokleby@uoit.ca \\ george.platanitis@uoit.ca
}

\begin{abstract}
Since 2005, with the endowment of the NSERC-GMCL Chair in Innovative Design Engineering at UOIT, and the Laptop-based, web-centric teaching approach, an ideal setting for the creation, prompt adoption, and implementation of advanced and innovative practices in teaching design engineering have been implemented, in addition to the use of traditional methods. A pilot program was recently completed to evaluate the use of Tablets in an engineering course. Tablets are currently used by faculty for teaching purposes at UOIT, but the program aims to integrate the use of Tablets within courses in the engineering design curriculum, namely for using CAD/CAM/CAE software. As CAD software capabilities improve, greater memory and computer speed is required, making the currently used conventional Laptops less useful for engineering design. In addition, Laptops do not lend themselves to graphical, free-form idea generation. It is intended that Tablets, with improved memory and processing speed, will facilitate CAD software usage, and hence, improve and enhance the overall design learning and application experience. Also, students can take advantage of software such as Microsoft OneNote to create preliminary sketches of designs and improve record-keeping of decisions during team meetings. In this pilot program, Tablet computers were issued to students and instruction personnel in a fourth-year Advanced Mechatronics course at UOIT. In this context, students were able to more efficiently carry out design assignments for term design projects, and students and instructors were able to evaluate the benefits of using Tablets. Overall, it was determined that Tablets were better as an engineering design tool compared to traditional Laptops.
\end{abstract}

\section{Introduction}

In recent years, the use of Tablet PCs (or Tablets) has been introduced into a wide range of academic courses at institutes around the world, from computer science, to humanities, to engineering. Although only a few experiences have been documented of curricular programs involving Tablets [1-5], in each case, the researchers attempted to identify merits for their particular courses, as well as areas that need to be improved upon in order to fully take advantage of the capabilities of the Tablet to enhance the classroom experience.

In one program [1], the authors reported on the use of first generation Tablet PCs, donated by Microsoft, in a first year computer science laboratory at Virginia Tech University. As part of a long term strategy, teaching laboratories were being evolved into highly flexible, adaptive spaces that would serve many teaching styles. Both lecture and lab rooms contained wireless support as well. For the lab sessions, both a traditional, desktop computer-equipped room and a non-traditional Tablet classroom were set up. Students were unaware of which of the lab sections were using the Tablets when they initially registered for their course. Students were allowed to bring wireless-enabled devices to lectures for the purpose of downloading starting materials for lecturing examples from the course website to be able to follow along, as well as try out their own ideas live during the class section. Lectures were taught as more discussionoriented sessions, where the instructor could demonstrate programming tasks live, with students watching as program codes were demonstrated and offering alternative solutions that could be tried on the spot. This methodology made lectures more engaging. Also, the wireless support allowed the instructor more freedom to move around the classroom and provide opportunities to involve students. In the lab sessions, students found the wireless enabled room equipped with Tablets advantageous, as they were allowed to move themselves in such a way with their Tablets to be able to work in groups. However, Tablets were found to be poor performers for some programming tasks. In particular, use of the pen as an input device for entering program text required significant corrective edits, greatly slowing the process of 
entering code, making it a poor match to entering and editing program text with the available generation of recognition software.

In another study, the authors examined the affect on learning using a wireless-enabled Tablet classroom at the School of Information Technology at Charles Darwin University, Australia [2, 3]. In [2], the authors framed their research project on the following learning principles - it is lifelong, occurs best in cultural context, is a social process, requires stimulation, and that direct experience helps shape a learner's understanding. The university received 45 Tablet PCs and associated wireless technology via a grant from Hewlett Packard, which were deployed into traditional lectures in three different ways - a database lecture to introduce JADE and OODBs, a web development lecture to teach accessibility principles (students took notes using digital ink), and a humanities lecture to fill an online student evaluation. Experience from this initial deployment led the authors to integrate lecture, lab, and tutorial into one 3-hour weekly workshop that allowed flexible time use to include some direct teaching, hands-on practice in collaborative groups, and problem solving discussions. Reactions to using the Tablets and the workshop mode were positive, showing an increase in quality and quantity of student discussions, and class dynamics changed such that there was an increase in focus on individual student work. In a related paper [3], the authors further discuss the deployment of Tablets using a first year programming course and a web development course as case studies, evaluating the implications of the workshop style of teaching and learning, as well as communities of practice, a concept describing how groups of people come together. Conditions in a Tablet-enabled classroom were found to be favourable in these contexts, where interactions between students and lecturer were found to have dramatically increased over traditional lecture/lab structure, as well as recognizing in one another each other's struggles, leading to increased participation in the workshop environment. As well, students were able to act as resources to each other, exchanging information and sharing ideas.

In [4], the use of wireless Tablets was evaluated for enhancing teaching and learning in engineering courses (Chemical, Electrical, and Computer Science). The Russ College of Engineering at Ohio University acquired twenty Gateway M280E Tablet PCs to be used in two electrical engineering, one chemical engineering, and one computer science course in Winter 2006, with these focus classes scheduled in rooms covered by wireless Internet access on campus without restrictions. Instructors developed teaching materials to take advantage of unique features of
Tablets. In a pre-deployment survey, students answered that they felt Tablets would improve their learning, being able to use the Tablet to take notes. Unexpectedly, students also answered that they would work on assignments unrelated to the focus classes. In the post-deployment survey, it was showed that students did find the Tablet useful for learning improvement, as well as being useful for Internet searches for material related to their classes. Students also felt that if they could keep their Laptops throughout the semester instead of signing them out for each class, as well as have a tutorial specific to the Tablet model, the initial student experience would be improved. Finally, regarding tool usage, students reported the need for more free time to learn Tabletspecific tools such as Microsoft Windows Journal.

While the above references show the potential for Tablets to be used in conjunction with available technology, such as wireless Internet access and specific tools to enhance learning in a blended learning approach in the classroom, the use of Tablets can be taken to another level, namely, using Tablets as part of Engineering Design Education. Few programs such as this exist. One such pilot program took place in the Department of Mechanical Engineering at Queen's University [5], where Tablets were introduced to students taking a capstone design course. One Tablet was given to each design team. One component that the Tablet replaced was the requirement of a hardcopy bound Design Notebook; students now were required to submit this Notebook electronically. Students used the Tablet to create design sketches, make notes and hand calculations (related to their designs using Microsoft OneNote), access web resources for parts, and add text notes to software analyses (ex., FEA) using the "translate" feature to convert handwriting to formal text. Overall, students found that the use of the Tablet improved recording of design decisions, allowed more engineering analysis throughout the term, and improved recording and sharing of meeting minutes. Students found the Tablet most useful during the early phases of design, and the wireless Internet access and portability of the machine allowed them to access manufacturing tips quickly, allowing them to update their solid models during manufacture. Tablet-based students were more likely to create and refine their analyses of their designs with the provided software as opposed to just noting the analysis. Although the speed of idea generation did not increase significantly compared to paper-based sketching, the Tablet allowed students to think more effectively during the concept sketching phase.

Herein, a pilot program in which Tablets were provided to every student in a fourth-year Advanced 
Mechatronics course at UOIT will be described. The goal was to assess the impact of Tablet use on design engineering. At the end of the semester, the pilot program was evaluated by surveying students and instructors.

\section{Advanced Mechatronics Course}

The Tablet pilot program was implemented in the fourth-year Advanced Mechatronics (ENGR4320U) course at UOIT. This course features an intensive hands-on project based learning approach structured around two design projects. In this course, students learn the tools required to design, model, analyse and control mechatronic systems. They model various system components in a unified approach and use tools for the simulation of the performance of these systems; characterize typical mechatronics systems in terms of their impacts on enhancement of performance, speed of operation, and physical size; and apply mechatronics to the robotics and automation industry, and other intelligent systems.

By the end of the course, successful students will be able to:

- Understand the basic principles involved in the design of mechatronic systems.

- Understand and deal with logic gates.

- Have a thorough knowledge of microprocessor architectures.

- Be able to deal with a variety of different types of sensors and actuators.

- Handle A/D and D/A conversion methods and implementation techniques.

- Understand and use the techniques of real-time multi-tasking.

- Able to apply programming concepts to mechatronic devices.

- Able to implement direct digital control techniques and equipment.

- Understand and use computer modeling and simulation techniques for mechatronic components.

Following are the general descriptions of the two design projects.

\subsection{Design Project 1}

For this project, students were responsible for designing and building an autonomous robot capable of following a path which the robot must sense (not have it preprogrammed) in an autonomous manner.
Students were required to demonstrate the functionality of their designs through a prototype demonstration. White tape on a dark floor marked the required path. The robot was required to follow the path until it reached a wall, and then reverse course along the same path. The robot design had to be robust for varying lighting conditions, self-contained, and fully autonomous. This hands-on experience allowed students the opportunity to learn how to interface actuators and sensors to a microcontroller, creating a mechatronic system that can be controlled. Students worked with a Mechatronics Design Kit which contained an $\mathrm{HC}(\mathrm{S}) 12$ microcontroller, three MicroMo motors, two Meccano Kits each with two motors, and various sensors and electrical components.

Deliverables for this project included a written report, oral presentation, prototype demonstration, and engineering documentation (fully dimensioned drawings, bill of materials, assembly drawings, etc.).

\subsection{Design Project 2}

The second project required students to build a robot that was capable of inspecting welds on a pipe in an autonomous manner. Two prototype demonstrations were required. The pipe was dark in colour, with white tape used to simulate welds. Robots were required to be robust for varying lighting conditions and had to be self-contained. Design Project 2 was divided into two phases. For the first phase of the project, the robots were required to locate the welds and stop at each weld for 5 seconds before moving on to the next weld. For the second phase, the robot had to inspect the entire weld upon reaching it, and identify any flaws; that is, the robot had to somehow be able to inspect around the pipe while following the weld, until it detects a flaw. If there was no flaw, the robot would then search for the next weld. When a flaw was found, the robot had to indicate that it had detected a flaw by lighting up a light emitting diode

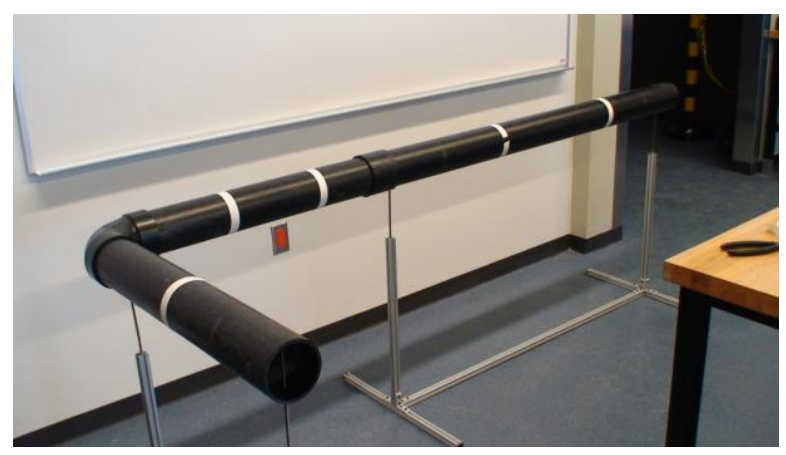

Figure 1. Pipe inspection test facility. 

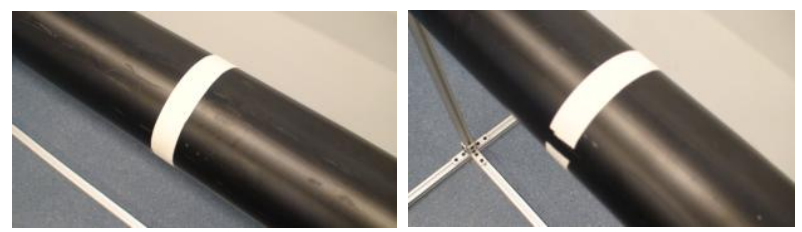

Figure 2. Simulated weld on the pipe (left) and simulated flaw in a weld (right).

(LED). Welds could have single, multiple, or no flaws. Figures 1 and 2 show the pipe used and the representation of welds and weld flaws.

Deliverables for each phase of this project included a written report, oral presentation, prototype demonstration, and engineering documentation.

\subsection{Tablet Use}

For Design Project 1, students used their conventional Laptops (Lenovo T61) to perform all the design activities necessary ${ }^{l}$. Software available included CodeWarrior (for programming the microprocessor board - students were allowed to use $\mathrm{C} / \mathrm{C}++$ or Assembly language); MATLAB with SIMULINK, and LabVIEW (for simulation of controllers); OrCAD with PSpice (for circuit design and analysis); and NX5, NX NASTRAN, and MSC Adams for CAD and CAE tasks.

For Design Project 2, students were issued Toshiba M700 Tablet Computers containing all the necessary design, engineering, analysis, and simulation software (as listed for the first project) to complete the second project. They were encouraged to use the unique features of the Tablet to help them in their mechatronic system design.

\section{Evaluation of Tablet Use}

\subsection{Laptop Survey Results}

For each of the two projects, students were evaluated on the effectiveness of using either a Laptop or a Tablet computer in their design projects. The questions used paralleled those used in the pilot study at Queen's University [5]. For the first design project, students were asked for their opinions on the effectiveness of using the Laptop for various stages and aspects of the design process. The following eight questions were asked, for which students responded to the degree that they agreed with each statement:

$1 \quad$ UOIT is a fully Laptop university with every student leasing a standard Laptop computer equipped with a suite of program specific software.
Q1 - A Laptop PC is an effective tool for capturing the results of brainstorming sessions.

Q2 - A Laptop PC encourages students to sketch ideas.

Q3 - A Laptop PC is an effective tool for creating sketches.

Q4 - A Laptop PC is an effective tool for sharing design ideas amongst group members.

Q5 - A Laptop PC is an effective tool for providing feedback on ideas to group members.

Q6 - A Laptop PC is more effective as a design notebook than a hardcopy notebook.

Q7 - A Laptop PC is an effective tool for documenting all stages of the design process.

Q8 - A Laptop PC is an effective tool for marking up drawings.

Agreement levels were any of Strongly Disagree (SD), Disagree (D), Neutral (N), Agree (A), and Strongly Agree (SA). Results of the first survey are presented in Figure 3. While students in general agreed that a Laptop is useful for the course design projects, large numbers of students indicated through this survey that the Laptops are not very useful when it comes to brainstorming and sketching ideas. In such a case, students would have been resorting to paper sketches and design notes in these preliminary stages, as a conventional Laptop does not have the necessary hardware or software to facilitate sketching and note taking (approximately 27 percent of students disagreed with Q1, 15 percent disagreed and 27 percent strongly disagreed with Q2, and 15 percent disagreed and 24 percent strongly disagreed with Q3). From Q4 through Q8, the general trend shows that students felt

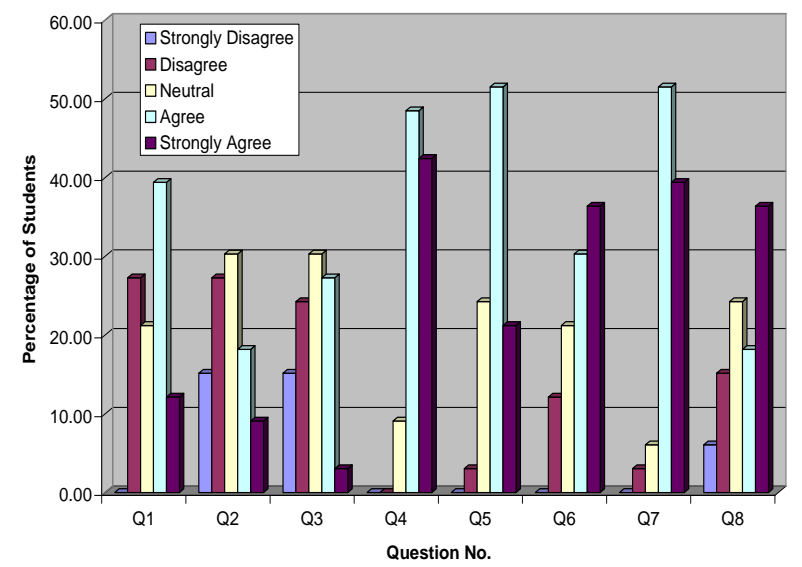

Figure 3. Distribution of responses of students to preliminary survey for Design Project 1 in using a Laptop as a design tool. 
that a Laptop was effective for the said aspects of the engineering design process.

In the survey, students also provided written comments regarding their overall experience with the Laptops as design tools. A few example comments follow:

"I think the laptops are useful tools, but I think tablets would be far more useful for sketching and sharing concepts. I think the school should replace most of the printers with scanners and the majority of the work and submissions should be digital."

"Not all software installed is useful or needed, thus causing the laptop to slow down...sketching tools are not available on the laptop except "Paint". Solid $3 D$ modeling programs are not fully licensed, which reduces effectiveness."

"Although there are drawbacks to having the laptop, the overall effect gives us the opportunity to explore various passages of interest (internet, software programs) to have a better final project."

"The laptop is good for organization of ideas and final copies of projects. Any idea generation is difficult and time consuming on the laptop. Same goes for any calculations that need to be done."

Overall, students found Laptops useful, but pointed out drawbacks, such as slowness, unnecessary software, and difficulties with generating sketches and preliminary ideas. They in turn suggested that Tablets may improve on these drawbacks.

\subsection{Tablet Survey Results}

For the second design project, students used the Tablet for various aspects of their design project. They were then given the same survey questions as before (with "Laptop" now replaced by "Tablet"), supplying answers as to the level they agreed with each statement. The results of the students' responses are shown in Figure 4. Compared to the results of the Laptop survey, students showed general agreement with the statements of Q4 through Q8 in using the Tablet, just as they did with the Laptop. However, the trend was different for Q1 through Q3, where students expressed agreement in that the Tablet was useful as a sketching tool (both creating and encouraging sketching of ideas), as well as to bringing together brainstorming ideas. No student disagreed with any of the statements of Q1 through Q3. Some example comments from students are presented below regarding the Tablet use:
"Good resource for brainstorming and sketching."

"I would have been glad if we had the chance to use this Tablet PC when we were in $3^{\text {rd }}$ year. Still, it's ok. It is an amazing tool for designing and evaluating."

"A little more awkward to use as a regular computer, but as an engineering tool, it is better."

"The tablet loses effectiveness as a design tool when it comes to modeling because of the small screen size."

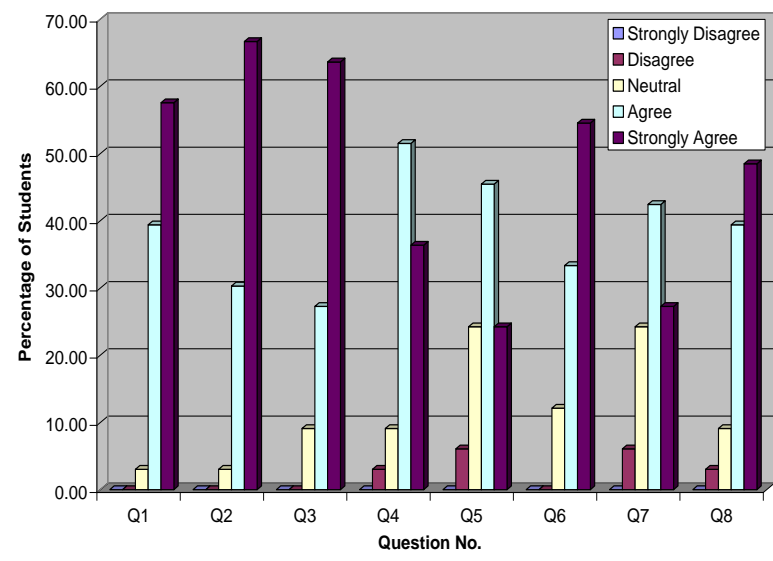

Figure 4. Distribution of responses of students to survey for Design Project 2, Phase 1, in using a Tablet PC as a design tool.

Students found the Tablet useful in a number of facets as a design tool, although some of the complaints arose from the small screen size, particularly for using CAD software. Also, some students commented on the lack of (adequate) sketching programs (these students may not have been aware that Microsoft OneNote was installed), and even searched for and downloaded alternative software, such as Alias Sketchbook Pro (for which a trial version is available online).

\section{Conclusions}

The use of Tablets in an academic setting is one that is well documented, where many of the features and capabilities otherwise not available with a conventional Laptop have been taken advantage of. A pilot project was implemented in the fourth-year Advanced Mechatronics Course at UOIT, where students made use of Tablets during a part of their term design project over the second half of the Winter 
2008 term. As part of the evaluation process of Tablet use, students answered surveys in which they rated different aspects of both a conventional UOIT issued Laptop and the Tablet, giving their impressions of the usefulness of both types of computers as engineering design tools for the term project. Survey results revealed some drawbacks of using a conventional Laptop. In particular, students found the Laptop inadequate for brainstorming, sketching, and organizing preliminary ideas, as the Laptop lacked the necessary hardware and software that would make this process more efficient. However, when students switched over to using the Tablet, they were better able to brainstorm, sketch, and organize ideas, as well as share them between their group peers. Also, students took advantage of opportunities to explore additional software, including Alias Sketchbook Pro, to have available at their disposal additional sketching programs. Overall, it was felt that the Tablet was better as an engineering design tool compared to the Laptop.

In a future project, Tablets will also be used in conjunction with recently donated 3DConnexion SpaceNavigator mice, which work with CAD software in enhancing $\mathrm{CAD}$ model views by allowing the user to zoom, pan, and rotate the models more efficiently.

\section{Acknowledgements}

The authors would like to thank the Natural Sciences and Engineering Research Council (NSERC) of Canada, General Motors Canada Limited (GMCL), and UOIT for financially supporting UOIT's Design Chair budget, part of which supported this research work. The authors are also grateful for the hardware and software provided by Partners for the Advancement of Collaborative Engineering Education (PACE). Finally, the authors would like to thank the Winter 2008 ENGR 4320U: Advanced Mechatronics class for their feedback on Laptops and Tablets.

\section{References}

[1] S. H. Edwards and N. D. Barnett, "Experiences Using Tablet PCs in a Programming Laboratory," ACM Special Interest Group for Information Technology Education (SIGITE'04), Salt Lake City, UT, October 28-30, 2004.

[2] J. Tutty, B. White, and R. Pascoe, "Experiences from a Wireless-enabled Tablet Classroom," The Australian Computing Education Conference, 2005, Newcastle, Australia.
[3] B. White and J. Tutty, "What, No Lectures!: Experiences from a Blended Tablet PC Classroom," National UniServe Science Conference Proceedings, University of Sydney, Australia, September 28-30, 2005,. pp. 124-130.

[4] C. Liu and Valerie Young, "Student Self-Reported Usage of Wireless Tablet PCs in Classrooms," $36^{\text {th }}$ ASEE/IEEE Frontiers in Education Conference, San Diego, CA, October 28-31, 2006.

[5] K. Firth and B. Surgenor, "Impact of Tablet PCs on Creativity in Engineering Design Education," The Fourth CDEN International Conference on Design Education, Innovation, and Practice, Winnipeg, Manitoba, Canada, July 22-24, 2006. 